\title{
A Novel Three-Dimensional Beamforming Antenna Array for Wireless Power Focusing
}

\author{
Mohammad A. Safar and Ayman S. Al-Zayed \\ Department of Electrical Engineering, Kuwait University, P.O. Box 5969, 13060 Al-Safat, Kuwait \\ Correspondence should be addressed to Mohammad A. Safar; msafer10@hotmail.com
}

Received 21 June 2016; Revised 20 August 2016; Accepted 30 August 2016

Academic Editor: Jonathan N. Blakely

Copyright (c) 2016 M. A. Safar and A. S. Al-Zayed. This is an open access article distributed under the Creative Commons Attribution License, which permits unrestricted use, distribution, and reproduction in any medium, provided the original work is properly cited.

\begin{abstract}
An antenna array capable of focusing the power transmitted to a specified point in space is modeled and simulated. This array will serve best for wireless power transmission applications where one of the goals is to maximize the power transfer efficiency. The array consists of 100 dipole antennas with each antenna transmitting a sum of 50 signals where each signal has a different frequency. This difference in frequency gives an additional degree of freedom that allows the overall beam pattern to be focused to a point in space instead of just a direction. The same array structure is also capable of transmitting power to multiple points in space which is promising when it comes to powering multiple points of interest.
\end{abstract}

\section{Introduction}

Antenna arrays are considered to be an important building block in many wireless applications such as radars, microwave imaging, satellite, and wireless communications. Arrays have many advantages such as the ability to transmit and/or receive signals to/from desired directions while maximizing signal-to-noise ratio, thus optimizing the overall power gain. In recent years, wireless power transfer applications have emerged and the use of antenna arrays in this area proved beneficial as seen in $[1,2]$. Wireless power transfer is accomplished in two ways: the first method is magnetic field resonance for near field applications [3-6] and the second method is electromagnetic radiation for far field applications $[1,2]$.

The basic idea in the near field approach is that the transmitter and receiver are linked through a loosely coupled pair of inductors and so power is transferred via the mutual inductance between these coils. The disadvantage of this approach is that the power transfer efficiency is greatly affected by the distance between the coils. There are many techniques to achieve larger transmission range. In [3], an intermediate coil is used to improve the power transfer efficiency. The power transfer efficiency in [4] is improved for distances greater than the strongly coupled region by using a modified frequency tracking method in which the frequency of the transmitting antenna or coil is tuned such that there is resonance between the transmitter and receiver. In addition, there is a complex load matching at the distance of interest to increase the range of efficient power transfer. The transmitter in [5] is made up of a three-element array of loops which resulted in increasing the coupling coefficient between the transmitter and receiver when compared to a single loop, hence increasing the power transfer efficiency. The characteristics of the transmitting coil or antenna must also be taken into account to optimize the power transfer efficiency. In [6], a planar near field plate fabricated on a circuit board is designed to shape and focus the beam for achieving higher resolution. To maximize the efficiency of power transfer in moving devices, the concept of resonators array was proposed in [7].

For far field applications, the power transfer efficiency is usually less than that of the near field especially if a single antenna is used as a transmitter. The power transfer efficiency in far field applications can be increased by using antenna arrays. In [2], an antenna array was used to optimize power transfer efficiency by adjusting the weights of the individual elements. Another advantage of using arrays is that they 
can be utilized to steer the transmitted power to a desired direction and to minimize sidelobes and hence the power radiated to unwanted directions is reduced [8]. In [9], a linear antenna array was developed to focus the power density to a desired point in space, where each antenna element transmits a pulse-like wave with specific time delays to accomplish focusing. The resulting power density has a local maximum at the desired point; however, other maxima are present in other locations as result of realization constraints.

In this paper, a new beamforming technique is introduced that is capable of transmitting power to a specified point in space, rather than a direction. This is achieved by multifrequency signaling technique which implies that each antenna element sends a number of signals each at a different frequency. By doing so, a third degree of freedom is added that allows control of the main beam in the radial direction and hence beamforming to a specific point in space is achieved. In this way, the antenna array directivity is increased compared to a regular directional antenna array. The multifrequency signaling antenna array is also capable of transmitting power to multiple points in space simultaneously. One of the advantages of using this technique is the fact that the power surrounding the point of interest is minimized. This is important for health and safety when considering indoor/outdoor wireless power transmission.

The paper is organized as follows: in Section 2, the concept of the proposed antenna array technique is given. In this section, how the basic theory, which only allows 2dimensional beam steering, can be modified such that the array can focus the beam to a point in space will be shown. Simulation results are given in Section 3 that will demonstrate the ability of the array to focus the beam to a desired point in space given by $\left(r_{o}, \theta_{o}\right.$, and $\left.\phi_{o}\right)$ in spherical coordinates. Finally in Section 4, the conclusion of this work is given.

\section{Using the Multifrequency Focusing Theory}

When a conducting element carries a time variant sinusoidal current density $\vec{J}(r)$, an electromagnetic field is created. The geometry of the current density dictates the form of the radiation vector $\vec{F}$ which is given by [10] as

$$
\vec{F}=\int e^{j \vec{k} \cdot r^{\prime}} \vec{J}\left(r^{\prime}\right) d^{3} r^{\prime}=F_{r} \widehat{r}+F_{\theta} \widehat{\theta}+F_{\phi} \widehat{\phi}
$$

where $j$ is $\sqrt{-1}, k$ is the wavenumber, and $r^{\prime}$ is a general representation of the dimension of the element. Moreover, $k=\omega \sqrt{\mu_{o} \epsilon_{o}}$ where $\omega$ represents the frequency of the transmitted waves in rad/s, $\mu_{o}$ is the permeability of free space, and $\epsilon_{o}$ is the permittivity of free space. It is clear from (1) that the radiation vector can have components in all 3 directions of the spherical coordinate system. When considering (1) for $N+1$ elements, distributed as shown in Figure 1, an antenna array is formed. Due to the physical separation between the elements, the total radiated electromagnetic field is the sum of $N+1$ time shifted (or time delayed) signals. This delay in time is utilized to control array's main beam characteristics as well as the direction of the beam.

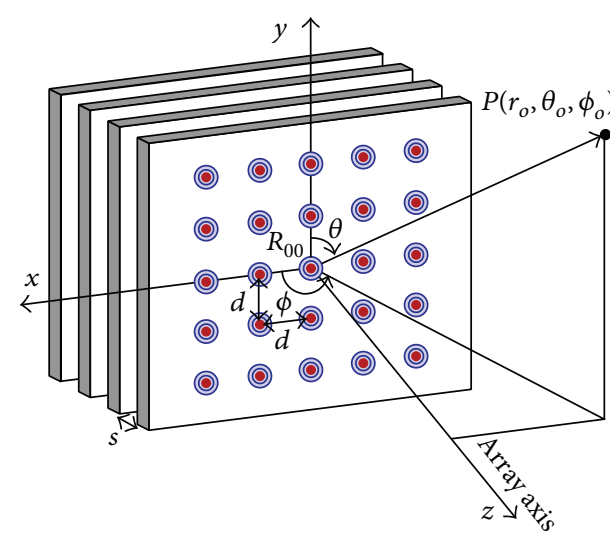

Radiator

Figure 1: Antenna array geometry for the three-dimensional array.

In the frequency domain, the control on the radiated pattern is accomplished by considering the array pattern given by [10]

$$
A(\vec{k})=\sum_{n=0}^{N} a_{n} e^{j \vec{k} \cdot \vec{d}_{n}}=A(\theta, \phi),
$$

where $a_{n}$ represents the weight (i.e., magnitude and phase) of the $n$th current signal, $\vec{k}$ is the wavenumber vector, and $\vec{d}_{n}$ is the location vector of the $n$th element.

The previously mentioned (1) and (2) are the classical picture of antenna array theory, where the resulting array factor is a function of $\theta$ and $\phi$ only. This implies that the direction of the electric field, hence the radiated power, can be controlled by using standard beamforming technique. This comes from the fact that the frequency of operation $\omega$ is fixed for all antenna elements and therefore the term $e^{-j k r}$ can be taken as a common factor; hence the array factor is independent of $r$. Moreover, in traditional antenna array theory, the time dependent factor, $e^{j \omega t}$, is dropped from the expressions of the electric and magnetic field as long as the frequency of operation is identical for all elements.

In this paper, a new technique that implements multiple frequency focusing is introduced. In this technique, each antenna element transmits a number of signals, $M+1$ signals, where each signal propagates at a different frequency depending on the incremental frequency $f_{s}\left(\omega_{s} / 2 \pi\right)$. All signals are centered around the fundamental frequency $f_{o}$ $\left(\omega_{o} / 2 \pi\right)$. The incremental frequency shift is $\omega_{s}$ which is very small compared to $\omega_{o}$. This shift or increment in frequency will affect the radiation vector and the wavenumber $k$, and therefore there will be $M+1$ radiation vectors. The $m$ th radiation vector will be

$$
\vec{F}_{m}=\int e^{j \vec{j}_{m} \cdot r^{\prime}} \vec{J}_{m}\left(r^{\prime}\right) d^{3} r^{\prime}=F_{r m} \widehat{r}+F_{\theta m} \widehat{\theta}+F_{\phi m} \widehat{\phi} .
$$

The new radiation vector given in (3) will give rise to a new array factor. Hence (2) must be updated to account for this change. Equation (2) is summed for $M+1$ frequencies by 
taking into account the time dependence. The wavenumber is then split as follows:

$$
k_{m}=\frac{\omega_{m}}{c}=\frac{\omega_{o}+m \omega_{s}}{c}=\frac{\omega_{o}}{c}=\frac{m \omega_{s}}{c}=k_{o}=m k_{s},
$$

where the speed of light $c=\sqrt{\mu_{o} \epsilon_{o}}$ and the array factor for the new antenna array is given by the following equation:

$$
\begin{aligned}
\vec{A}(\vec{k}, t) & =\vec{A}(r, \theta, \phi, t) \\
& =\sum_{m=0}^{M} \sum_{n=0}^{N} a_{m n} k_{m} e^{j m \omega_{s} t} e^{j \vec{k}_{m} \cdot \vec{d}_{n}} e^{-j m k_{s} r}\left[F_{\theta m} \widehat{\theta}+F_{\phi m} \widehat{\phi}\right] .
\end{aligned}
$$

The multiple frequency signaling resulted in having the radial distance $r$ and the time $t$ dependent exponentials inside the summation and hence the array factor in (4) will now be dependent on $r$ and $t$.

Note that the split in the wavenumber is only considered for the radial distance and time exponentials while the exponentials that hold angular and position characteristics are not affected. This is due to the fact that multifrequency signaling has negligible effect on the angular part of the total electric field. This will be seen in Section 3 where the simulation results are discussed.

The antenna elements are distributed in a 3-dimensional array as shown in Figure 1. The figure shows how the elements are distributed on one layer only, which is on the $x y$-plane with element $R_{00}$ at the origin. The entire array consists of 4 identical layers stacked on top of each other and separated by a distance $s$. All elements (radiators) are identical dipole antennas with dimension $\lambda_{o} / 8=3.75 \mathrm{~cm}$ (where $\lambda_{o}=$ $c / f_{o}$ ) carrying a sinusoidal current and oriented in the same direction. The distance between any two adjacent radiators $d$ is $\lambda_{o} / 4$. In each layer, there are 25 elements and hence the total number of elements is 100 .

Dipole antennas are linear $z$-oriented antennas and therefore only the $F_{\theta}$ term of the radiation vector is present in (5). This implies that the array factor in (5) will become a scalar quantity.

The dot product in (5) between the wavenumber and the position vector is expanded to update the array factor to the antenna array geometry. Following the analysis in [10], the array factor becomes

$$
\begin{aligned}
& A(r, \theta, \phi, t)=\sum_{m=0}^{M} \sum_{n_{x}=1}^{5} \sum_{n_{y}=1}^{5} \sum_{n_{z}=1}^{4} a_{m n_{x} n_{y} n_{z}} \\
& \cdot k_{m} e^{j n_{x} k_{m} d \sin \theta \cos \phi} e^{j n_{y} k_{m} d \sin \theta \sin \phi} e^{j n_{z} k_{m} s \cos \theta} e^{-j m k_{s} r} e^{j m \omega_{s} t} F_{\theta m} .
\end{aligned}
$$

This new beamforming technique given in (6) includes weight coefficients $a_{m n_{x} n_{y} n_{z}}$ for each signal in each antenna. The desired electric field or power radiated is a delta function centered at a desired point in space given by $\left(r_{o}, \theta_{o}, \phi_{o}\right)$. To achieve this desired function, the weight coefficients need to be optimized. Since there is also a time dependence in the array factor, the optimized coefficients should be chosen with consideration of time dependency effect. The requirement in space and time can be achieved by finding the appropriate coefficient values that will result in

$$
\begin{aligned}
A(r, \theta, \phi, t)= & K_{o} \delta\left(\psi_{1}-\psi_{1 o}\right) \delta\left(\psi_{2}-\psi_{2 o}\right) \delta\left(\psi_{3}-\psi_{3 o}\right) \\
& \cdot \delta\left(\psi_{4}-\psi_{4 o}\right),
\end{aligned}
$$

where

$$
\begin{aligned}
& \psi_{1}=k r \\
& \psi_{2}=k d \sin \theta \cos \phi, \\
& \psi_{3}=k d \sin \theta \sin \phi \\
& \psi_{4}=k s \cos \theta \\
& \psi_{1 o}=k r_{o} \\
& \psi_{2 o}=k d \sin \theta_{o} \cos \phi_{o} \\
& \psi_{3 o}=k d \sin \theta_{o} \sin \phi_{o} \\
& \psi_{4 o}=k s \cos \theta_{o}
\end{aligned}
$$

where $K_{o}$ is the amplitude of the focused field. The four delta functions in (7) describe the shape of the desired field or power for the radial distance and angular direction. Applying the orthogonality property of exponentials presented in [10], the optimum coefficients for achieving (7) are given by

$$
\begin{aligned}
a_{m n_{x} n_{y} n_{z}}= & \left(\frac{e^{-j n_{x} k_{m} d \sin \theta_{o} \cos \phi_{o}} e^{-j n_{y} k_{m} d \sin \theta_{o} \sin \phi_{o}}}{16 \pi^{4} k_{m} F_{\theta m}}\right) \\
& \cdot\left(e^{-j n_{z} k_{m} s \cos \theta_{o}} e^{j m k_{s} r_{o}} e^{-j m \omega_{s} t}\right) .
\end{aligned}
$$

Note that in (9) the optimum coefficient is a function of time. This is necessary to cancel the time dependent exponential present in the array factor in (6) to make the desired beam pattern time independent as in (7). This can be achieved by using phase shifters that can continuously change the phase of the outgoing signals according to (9). Besides the time independency issue, other challenges need to be addressed in order to realize the proposed three-dimensional antenna array. First, the array requires antenna elements with flat frequency response over wide bandwidth. Another challenge is feeding these antennas with multiple frequencies and with the correct timing between the antenna elements.

The array can be used to transmit power to multiple points in the surrounding space. If the desired points in space were given by $\left(r_{o}, \theta o, \phi_{o}\right)$ and $\left(r_{p}, \theta_{p}, \phi_{p}\right)$, then the appropriate array factor takes the following form:

$$
\begin{aligned}
A(r, \theta, \phi, t)= & K_{o} \delta\left(\psi_{1}-\psi_{1 o}\right) \delta\left(\psi_{2}-\psi_{2 o}\right) \\
& \cdot \delta\left(\psi_{3}-\psi_{3 o}\right) \delta\left(\psi_{4}-\psi_{4 o}\right) \\
+ & K_{p} \delta\left(\psi_{1}-\psi_{1 p}\right) \delta\left(\psi_{2}-\psi_{2 p}\right) \\
& \cdot \delta\left(\psi_{3}-\psi_{3 p}\right) \delta\left(\psi_{4}-\psi_{4 p}\right)
\end{aligned}
$$


where

$$
\begin{aligned}
& \psi_{1 p}=k r_{p}, \\
& \psi_{2 p}=k d \sin \theta_{p} \cos \phi_{p}, \\
& \psi_{3 p}=k d \sin \theta_{p} \sin \phi_{p}, \\
& \psi_{4 p}=k s \cos \theta_{p},
\end{aligned}
$$

where $K_{p}$ is the amplitude of the second focused field corresponding to the point $\left(r_{p}, \theta_{p}, \phi_{p}\right)$. Once again if the orthogonality property of exponentials is applied, with the adaptation of continuous phase shifters, the antenna array factor becomes time independent, and the optimum coefficients for multiple transmissions will now be

$$
\begin{aligned}
& a_{m n_{x} n_{y} n_{z}}=a e^{-j m \omega_{s} t}, \\
& a=\frac{e^{-j n_{x} k_{m} d \sin \theta_{o} \cos \phi_{o}} e^{-j n_{y} k_{m} d \sin \theta_{o} \sin \phi_{o}} e^{-j n_{z} k_{m} s \cos \theta_{o}} e^{j m k_{s} r_{o}}}{16 \pi^{4} k_{m} F_{\theta m}} \\
& \quad+\frac{e^{-j n_{x} k_{m} d \sin \theta_{p} \cos \phi_{p}} e^{-j n_{y} k_{m} d \sin \theta_{p} \sin \phi_{p}} e^{-j n_{z} k_{m} s \cos \theta_{p}} e^{j m k_{s} r_{p}}}{16 \pi^{4} k_{m} F_{\theta m}} .
\end{aligned}
$$

In general, to transmit to many points in space $P$, the optimum coefficients are simply the superposition or summation of all sets of coefficients associated with each point in space. Hence the general solution for the optimum coefficients transmitting to $P$ points in space is as follows:

$$
\begin{aligned}
& a_{m n_{x} n_{y} n_{z}} \\
& =b \sum_{i=1}^{P} \frac{e^{-j n_{x} k_{m} d \sin \theta_{i} \cos \phi_{i}} e^{-j n_{y} k_{m} d \sin \theta_{i} \sin \phi_{i}} e^{-j n_{z} k_{m} s \cos \theta_{i}} e^{j m k_{s} r_{i}}}{16 \pi^{4} k_{m} F_{\theta m}}, \\
& b=e^{-j m \omega_{s} t}
\end{aligned}
$$

To examine the performance of the multifrequency signaling antenna array, the directivity is studied. The directivity of an antenna array is the radiation intensity towards a specific direction $\left(\theta_{o}, \phi_{o}\right)$ normalized by the intensity of an isotropic array. Since the multifrequency signaling antenna array can focus to a specific point in space the definition of the directivity is updated. In this case, the directivity is the radiation intensity towards a specific focus point in space $\left(r_{o}, \theta_{o}, \phi_{o}\right)$ normalized by the intensity of an isotropic array. The directivity is given as follows:

$$
D=\frac{\left|A\left(r_{o}, \theta_{o}, \phi_{o}\right)\right|^{2}}{\int_{0}^{r_{o}} \int_{0}^{\pi} \int_{0}^{2 \pi}|A(r, \theta, \phi)|^{2} \sin \theta d r d \theta d \phi}
$$

A comparison will be made between the multifrequency signaling antenna array with directivity $D_{\text {MFSAA }}$ and the typical directional antenna array with directivity $D_{\text {directional }}$. The comparison is necessary to show the advantage of focusing to a point in space rather than a direction. To make the comparison fair, both antenna arrays have identical geometrical structures and individual elements as shown in Figure 1. The only difference is that the multisignaling antenna array uses a number of signals per element while the directional antenna array uses one signal per element.

The array factor shown in (14) can be separated into two functions $R(r)$ and $\Omega(\theta, \phi)$ such that

$$
A(r, \theta, \phi)=R(r) \Omega(\theta, \phi),
$$

where $R(r)$ represents the radial distance dependence of the array factor and $\Omega(\theta, \phi)$ represents the angular dependence of the array factor. Both antenna arrays, the multi-frequency signaling and the directional, have the same angular dependence $\Omega(\theta, \phi)$. They only differ when considering $R(r)$. This implies that the ratio $D_{\text {MFSAA }} / D_{\text {directional }}$ can be written as

$$
\begin{aligned}
\frac{D_{\text {MFSAA }}}{D_{\text {directional }}}= & \frac{\left|R_{\text {MFSAA }}\left(r_{o}\right)\right|^{2}}{\int_{0}^{r_{o}}\left|R_{\text {MFSAA }}(r)\right|^{2} d r} \\
& \cdot \frac{\int_{0}^{r_{o}}\left|R_{\text {directional }}(r)\right|^{2} d r}{\left|R_{\text {directional }}\left(r_{o}\right)\right|^{2}}
\end{aligned}
$$

Since the directional antenna array has no radial distance dependence $R_{\text {directional }}(r)=1$, hence (16) simplifies to

$$
\frac{D_{\text {MFSAA }}}{D_{\text {directional }}}=\frac{r_{o}\left|R_{\text {MFSAA }}\left(r_{o}\right)\right|^{2}}{\int_{0}^{r_{o}}\left|R_{\text {MFSAA }}(r)\right|^{2} d r} \text {. }
$$

\section{Simulation Results}

The new multifrequency signaling antenna array is simulated. The total number of elements is 100 dipole antennas, each with a dimension of $3.75 \mathrm{~cm}$ and a separation between one element and its adjacent neighbors of $7.5 \mathrm{~cm}$. Each antenna transmits a sum of 50 signals, each having different frequency of operation. The frequency range is $1-1.75 \mathrm{GHz}$ and hence the frequency increment between adjacent frequencies $f_{s}$ is $15 \mathrm{MHz}$.

3.1. Focusing to a Single Point. Figure 2(a) shows the normalized array factor versus radial distance for a focused distance $r_{o}=5$ meters away from the antenna array and for a direction given by $\theta_{o}=\pi / 4\left(45^{\circ}\right)$ and $\phi_{o}=\pi\left(180^{\circ}\right)$.

Figure 2(b) shows the normalized array factor versus angular direction for a desired focus point given by $r_{o}=5 \mathrm{~m}$, $\theta_{o}=\pi / 4$, and $\phi_{o}=\pi$. It is clear from Figure $2(\mathrm{a})$ that, by implementing multifrequency signaling, the array factor becomes $r$-dependent and by judicious choice of the weight values (9), it is possible to focus the beam at the desired radial direction which in this case was 5 meters. Furthermore, Figure 2(b) shows how the array was able to direct the beam to the desired direction and have the capability of focusing the beam to the desired point in space with negligible power elsewhere.

\subsection{Effect of Frequency Increment on Resolution Performance.} Further simulations are carried out to study the effect of the frequency increment on the resolution of the focused beam. This approach requires a bandwidth of $750 \mathrm{MHz}$ (for 


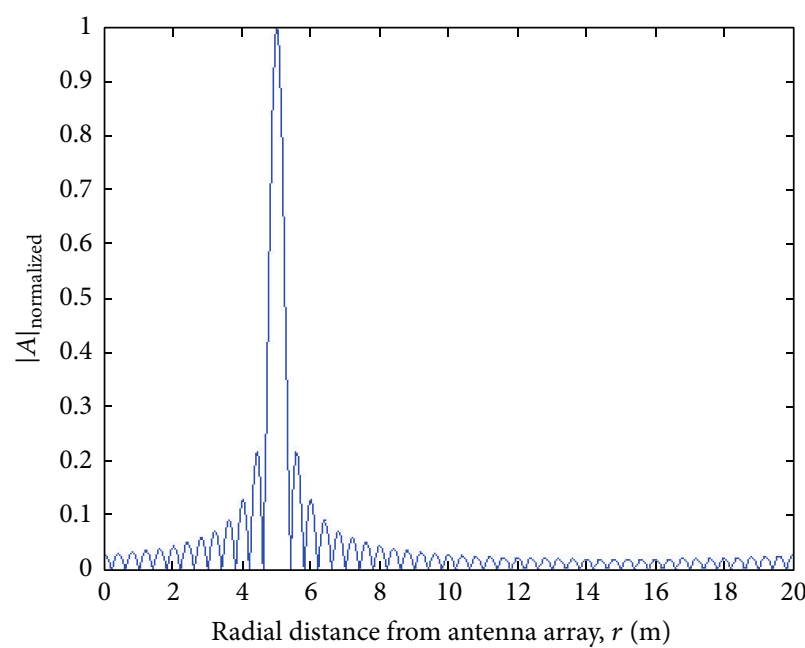

(a)

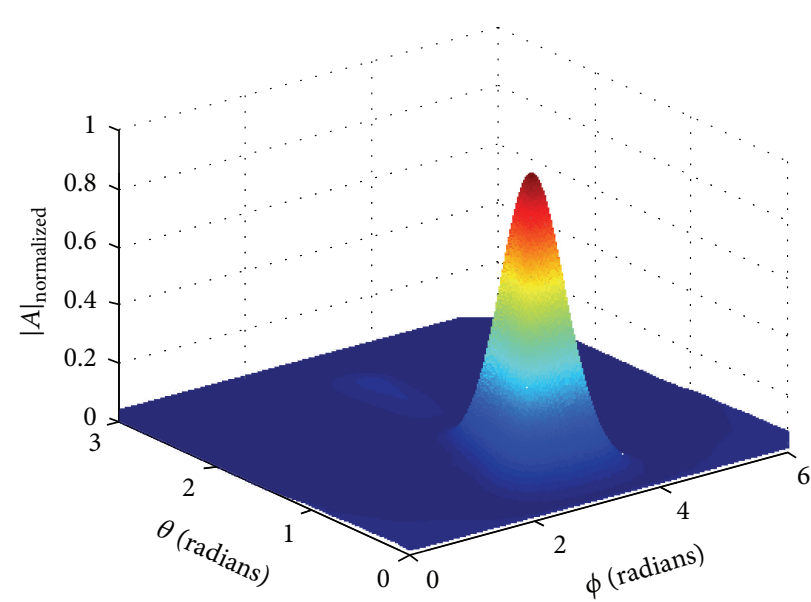

(b)

Figure 2: Normalized array factor for a desired focus point: $\left(r_{o}=5 \mathrm{~m}, \theta_{o}=\pi / 4\right.$, and $\left.\phi_{o}=\pi\right)$ (a) versus radial distance and (b) versus angular direction.

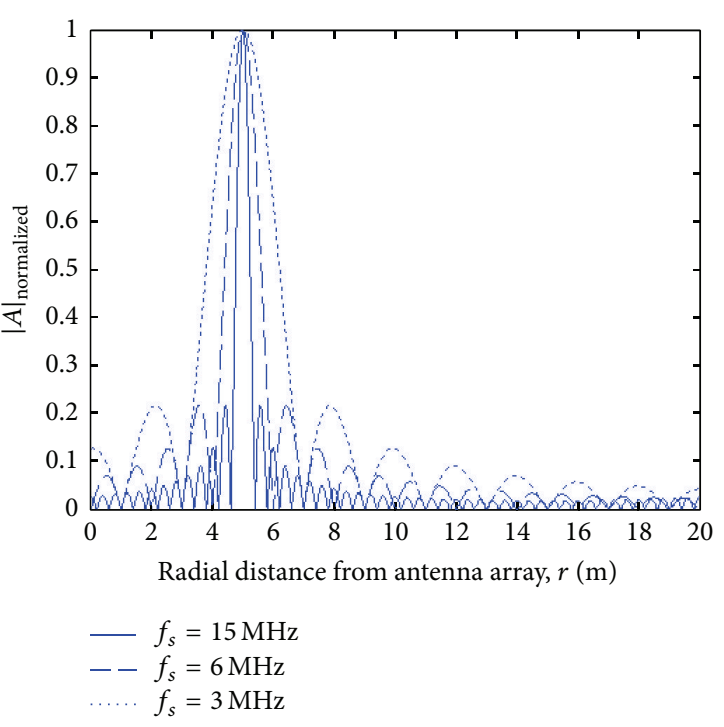

FIGURE 3: Normalized array factor versus radial distance for a desired focus point $\left(r_{o}=5 \mathrm{~m}, \theta_{o}=\pi / 4\right.$, and $\left.\phi_{o}=\pi\right)$ for three different frequency increments 15,6 , and $3 \mathrm{MHz}$.

a $15 \mathrm{MHz}$ frequency increment). In Figure 3, the normalized array factor versus radial distance for a desired focus point $(5 \mathrm{~m}, \pi / 4, \pi)$ for three different frequency increments 15,6 , and $3 \mathrm{MHz}$ is displayed.

It is evident from the figure that, by increasing the frequency increment, the radial resolution becomes sharper and therefore the wider the bandwidth used, the better the resolution of the beam. The sharpest resolution shown in the figure was for the $15 \mathrm{MHz}$ frequency increment and hence a $750 \mathrm{MHz}$ of bandwidth will be required. Wide bandwidth will require a receiver that has a flat amplitude and time delay response over the required bandwidth.
Figures 4(a) and 4(b) show how the resolution or concentration of beam varies with the frequency increment when considering the angular dependence of the array factor for increments of 15 and $3 \mathrm{MHz}$. By comparing Figures 4(a) and 4(b), it is evident that the angular resolution of the beam increases by increasing the frequency increment.

3.3. Effect of the Number of Signals on Resolution Performance. A study is conducted to examine the effect of the number of signals on the resolution performance. Figure 5 shows the normalized array factor versus radial distance for a desired focus point $(5 \mathrm{~m}, \pi / 4, \pi)$ for three different cases of number of signals 50,30 , and 20 while the incremental frequency is set at $15 \mathrm{MHz}$ for all simulations.

As seen in Figure 5, the resolution of the array factor in the radial direction improves and becomes more focused as the number of signals used increases. One can reduce the number of signals needed to be added to simplify the transmitter circuit and also to decrease the bandwidth of frequencies, since the bandwidth is directly proportional to the number of signals given by $M+1$.

To demonstrate the angular dependency of the array factor, Figure 6 shows the normalized array factor versus angular direction for a desired focus point $(5 \mathrm{~m}, \pi / 4, \pi)$ for the case of 20 signals. By comparing Figure 6 with Figure 4(a) (50 signals), it can be noticed that the higher the number of signals is, the better the resolution or focusing becomes.

3.4. Directivity. One important figure of merit is the directivity of an antenna array. Figure 7 shows how the ratio $D_{\text {MFSAA }} / D_{\text {directional }}$ given by (17) varies with the desired radial distance $r_{o}$. It is evident from Figure 7 that the multifrequency signaling antenna array is superior to the typical directional array when it comes to directivity, especially when considering desired radial distances that are far away from the array structure. This shows that focusing the beam to a point 


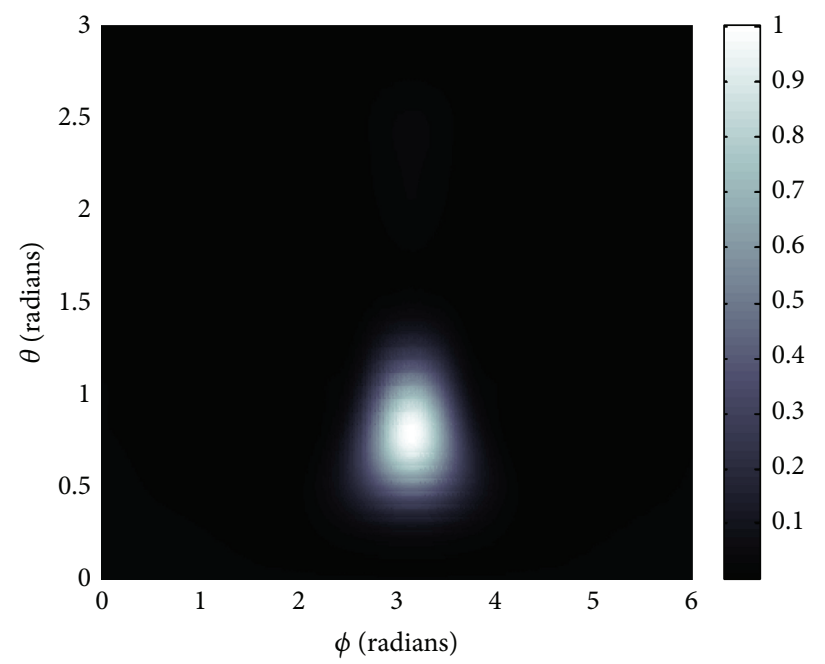

(a)

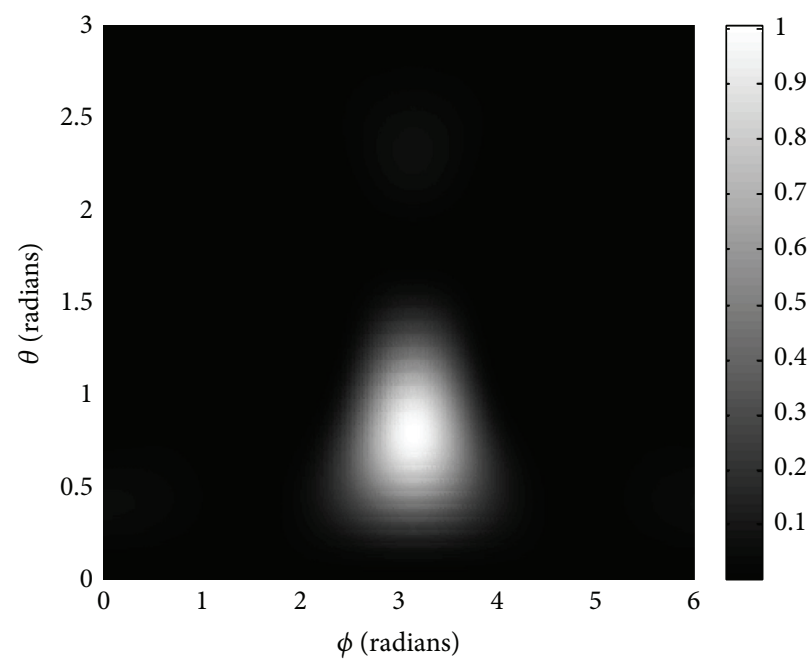

(b)

FIGURE 4: Normalized array factor versus angular direction for a desired focus point $\left(r_{o}=5 \mathrm{~m}, \theta_{o}=\pi / 4\right.$, and $\left.\phi_{o}=\pi\right)$ for a frequency increment of (a) $f_{s}=15 \mathrm{MHz}$ and (b) $f_{s}=3 \mathrm{MHz}$.

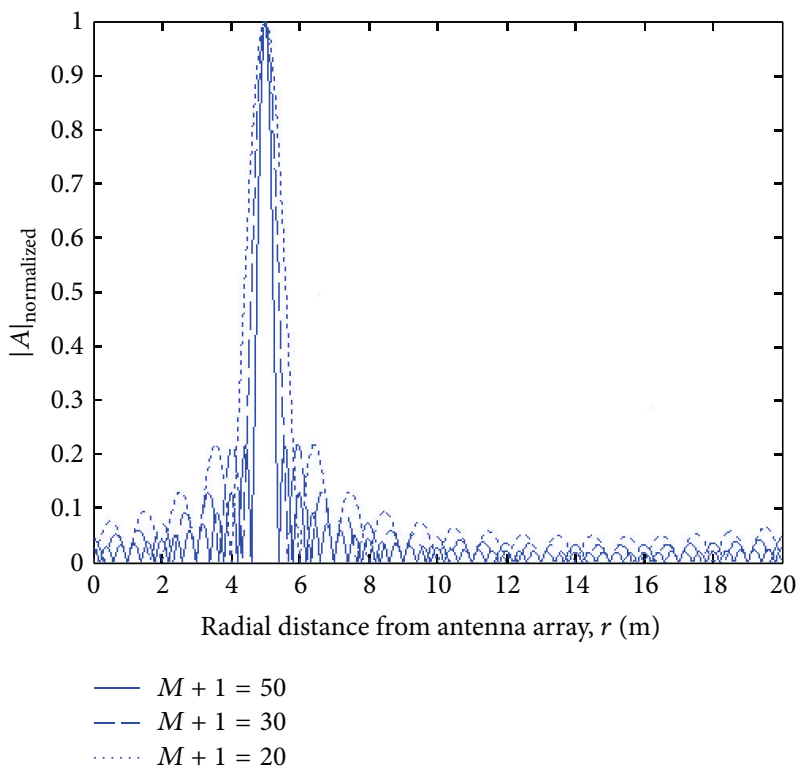

FIGURE 5: Normalized array factor versus radial distance for a desired focus point $\left(r_{o}=5 \mathrm{~m}, \theta_{o}=\pi / 4\right.$, and $\left.\phi_{o}=\pi\right)$ for three different numbers of signals 50,30 , and $20\left(f_{s}=15 \mathrm{MHz}\right)$.

in space is much more directive than steering to a certain direction in space.

3.5. Focusing to Multiple Points. To demonstrate the ability of the proposed multifrequency signaling antenna array to transmit to multiple points in space, (12) will be used to find appropriate values for the coefficients to focus the power to two different points simultaneously. Figure 8(a) shows the normalized array factor versus radial distance for two desired focus points $(5 \mathrm{~m}, \pi / 4, \pi)$ and $(8 \mathrm{~m}, \pi / 2, \pi / 2)$. Figure 8 (b) shows the normalized array factor versus angular direction. It is

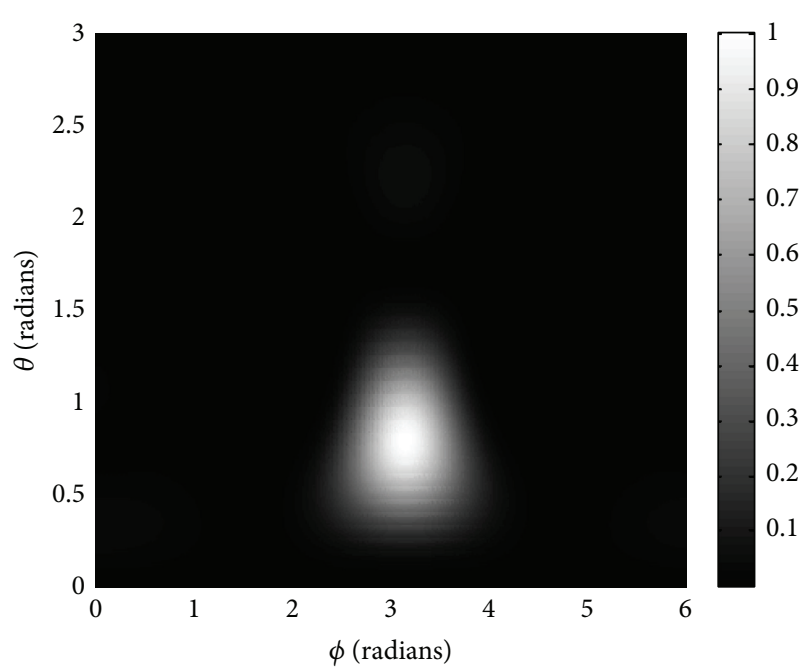

FIgURE 6: Normalized array factor versus angular direction for a desired focus point $\left(r_{o}=5 \mathrm{~m}, \theta_{o}=\pi / 4\right.$, and $\left.\phi_{o}=\pi\right)$ for 20 signals $\left(f_{s}=15 \mathrm{MHz}\right)$.

clear from the figures that the array has the ability to focus to two different points simultaneously. From the previous two studies on parameter effect (frequency increment and number of signals) on the beam resolution, it can be deduced that based on the system requirement these parameters can be determined accordingly.

\section{Conclusion}

The theory of a multifrequency signaling antenna array capable of transmitting electromagnetic field or power to a specific single or multiple points in space has been presented. The three-dimensional beamforming or focusing was achieved by applying multifrequency signaling in which each element in 


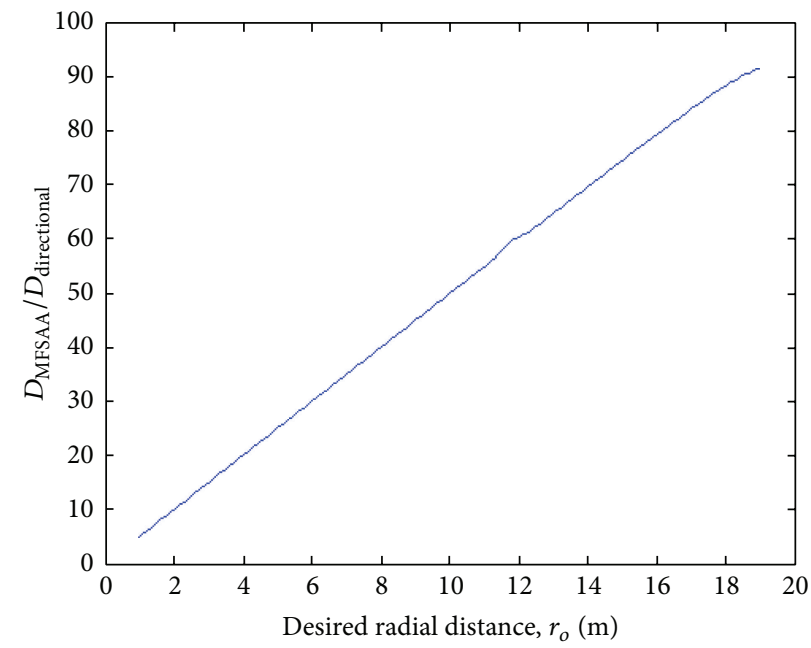

FIGURE 7: The ratio $D_{\text {MFSAA }} / D_{\text {directional }}$ versus desired radial distance.

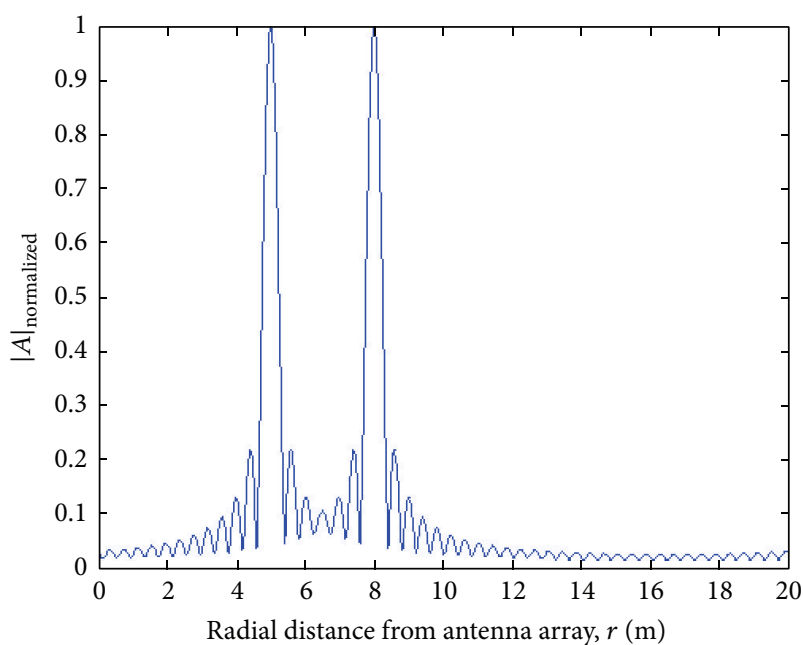

(a)

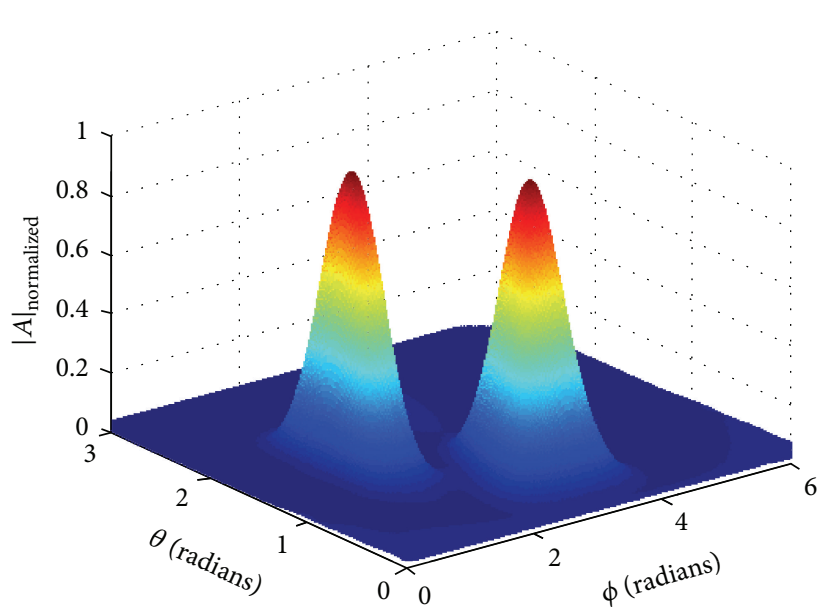

(b)

FIGURE 8: Normalized array factor for desired focus points $\left(r_{o}=5 \mathrm{~m}, \theta_{o}=\pi / 4\right.$, and $\left.\phi_{o}=\pi\right)$ and $\left(r_{p}=8 \mathrm{~m}, \theta_{p}=\pi / 2\right.$, and $\left.\phi_{p}=\pi / 2\right)(\mathrm{a})$ versus radial distance and (b) versus angular direction.

the array transmits a number of sinusoidal signals with different frequencies. This frequency increment adds a third degree of freedom in the array factor which in turn gives the array the ability to control the power transmitted in both radial distance and angular direction. Simulations demonstrated the ability of the array to focus the beam to a specific point while minimizing power transmission elsewhere. Directivity of the multifrequency signaling antenna array was superior to the typical directional antenna array. Moreover, it was demonstrated that the array is capable of transmitting to two points in space simultaneously.

Multifrequency signaling antenna array utilizes a discreet number of frequencies, instead of one frequency of operation. A study was conducted on the effect of bandwidth or frequency increment between signals on the beam resolution. It was found that as the frequency increment or bandwidth was increased the resolution of the beam became shaper. Another study was conducted to investigate the effect of the number of signals used on the beam resolution. It was found that as the number of used signals increased, the beam resolution improves or gets sharper. Therefore, desired system requirement can be determined based on selecting the studied parameters as mentioned earlier. This technique is promising for future wireless power transmission applications.

\section{Competing Interests}

The authors declare no competing interests regarding the publication of this paper.

\section{References}

[1] Y. Li and V. Jandhyala, "Design of retrodirective antenna arrays for short-range wireless power transmission," IEEE Transactions on Antennas and Propagation, vol. 60, no. 1, pp. 206-211, 2012. 
[2] G. Oliveri, L. Poli, and A. Massa, "Maximum efficiency beam synthesis of radiating planar arrays for wireless power transmission," IEEE Transactions on Antennas and Propagation, vol. 61, no. 5, pp. 2490-2499, 2013.

[3] J. Kim, H.-C. Son, K.-H. Kim, and Y.-J. Park, "Efficiency analysis of magnetic resonance wireless power transfer with intermediate resonant coil," IEEE Antennas and Wireless Propagation Letters, vol. 10, pp. 389-392, 2011.

[4] J. Park, Y. Tak, Y. Kim, Y. Kim, and S. Nam, "Investigation of adaptive matching methods for near-field wireless power transfer," IEEE Transactions on Antennas and Propagation, vol. 59, no. 5, pp. 1769-1773, 2011.

[5] D. Williams and M. Manteghi, "Reactive near field magnetic coupling utilizing a staggered phased array," in Proceedings of the IEEE Antennas and Propagation Society International Symposium (APSURSI '11), pp. 2453-2456, July 2011.

[6] M. F. Imani and A. Grbic, "Design of a planar near-field plate," in IEEE Antennas and Propagation Society International Symposium, p. 1, 2012.

[7] B. Wang, D. Ellstein, and K. H. Teo, "Analysis on wireless power transfer to moving devices based on array of resonators," in Proceedings of the IEEE 6th European Conference on Antennas and Propagation (EuCAP '12), pp. 964-967, IEEE, March 2012.

[8] E. E. Hassan and H. A. Ragheb, "Sidelobes level reduction using spatial optimization of the array factor," IEEE Antennas and Wireless Propagation Letters, vol. 11, pp. 756-759, 2012.

[9] H. J. Visser, "Maximizing DC power in WPT using a transient transmit array antenna," in Proceedings of the IEEE Wireless Power Transfer Conference (WPTC '16), pp. 1-4, Aveiro, Portugal, May 2016.

[10] S. J. Orfanidis, Electromagnetic Waves and Antennas, Rutgers University, 2002, http://www.ece.rutgers.edu/ orfanidi/ewa/. 


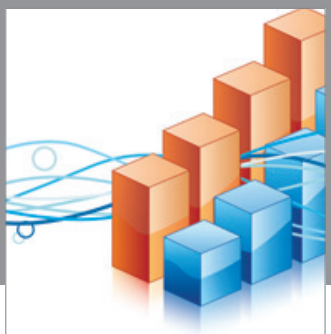

Advances in

Operations Research

vatem alat4

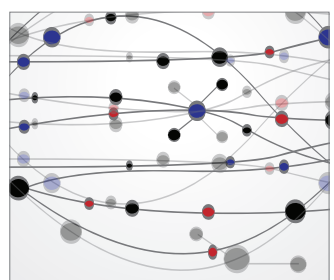

\section{The Scientific} World Journal
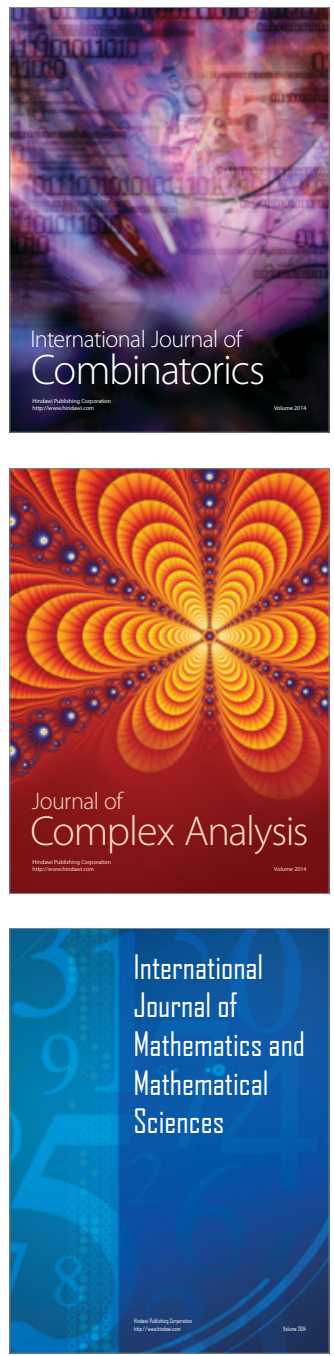
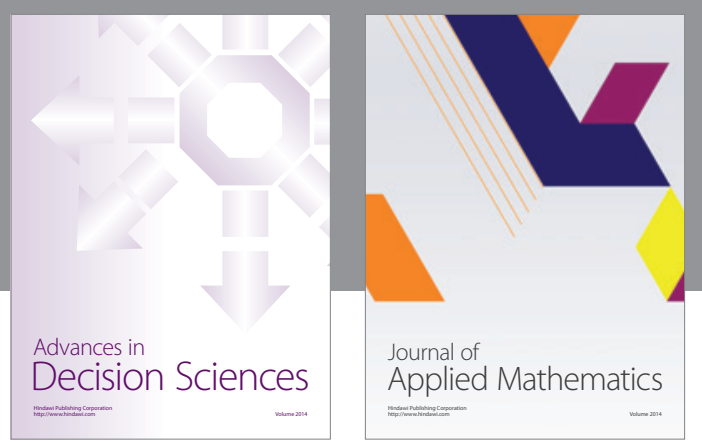

Algebra

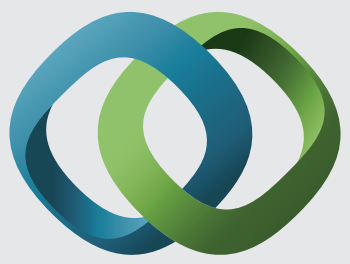

\section{Hindawi}

Submit your manuscripts at

http://www.hindawi.com
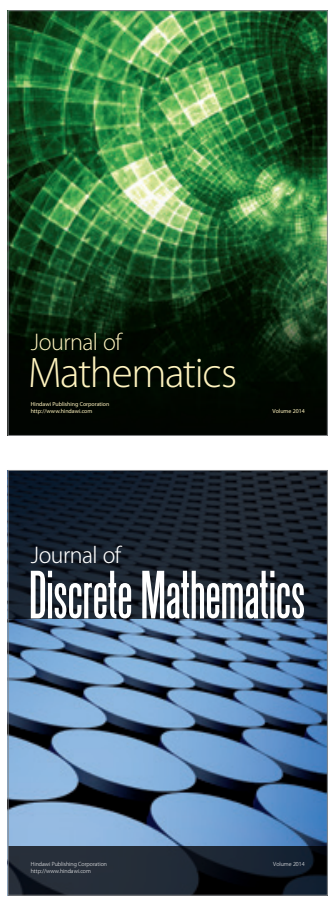

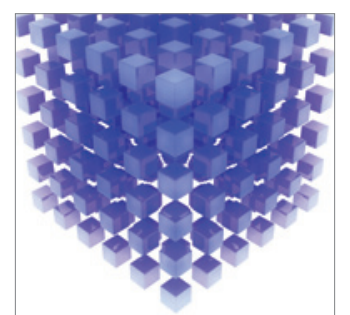

Mathematical Problems in Engineering
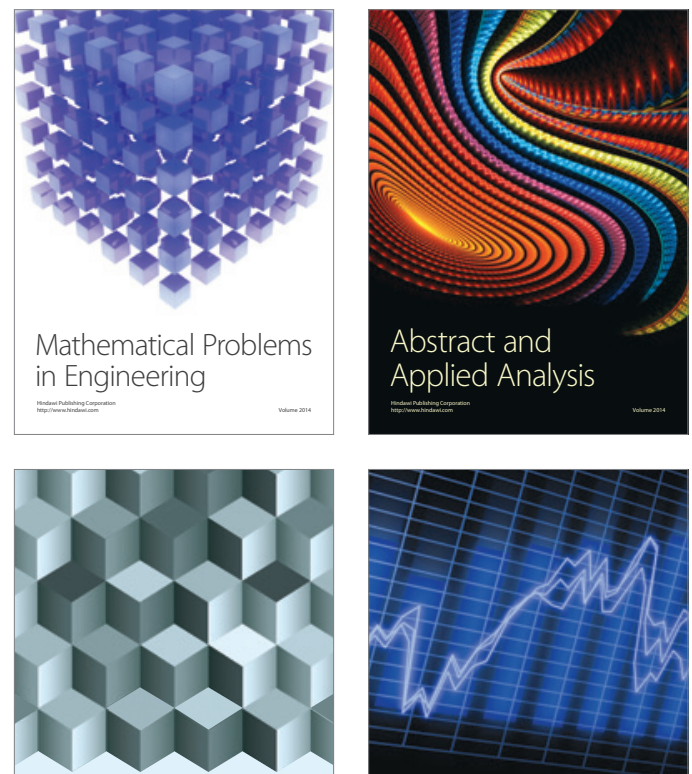

Journal of

Function Spaces

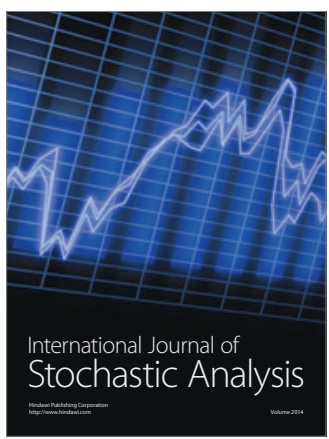

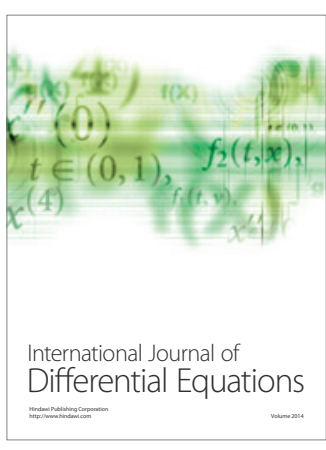
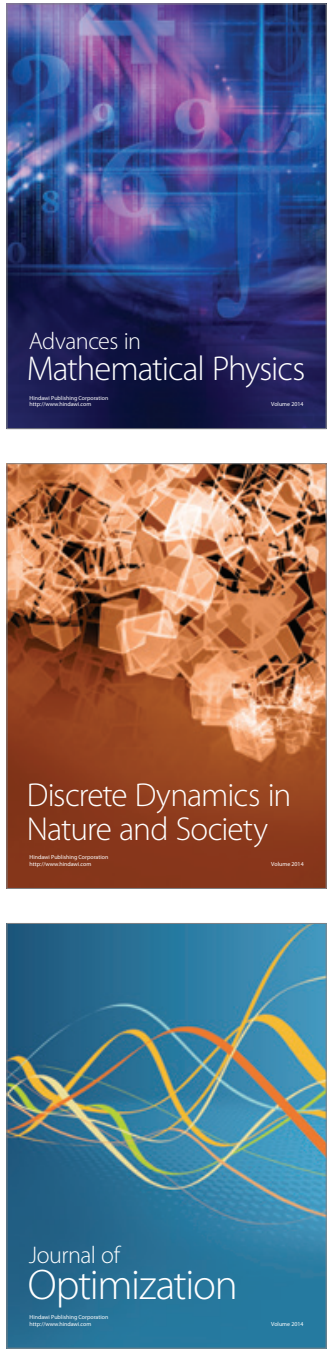